\title{
Desafios do enfermeiro do Centro Cirúrgico frente à pandemia da COVID-19 e transição de uma sala cirúrgica em unidade de terapia semi-intensiva
}

Challenges for operating room nurses facing the COVID-19 pandemic and the transition from an operating room to a semi-intensive care unit

Retos del enfermero del centro quirúrgico ante la pandemia del COVID-19 y transición del centro quirúrgico a una unidad de cuidados semi-intensivos

Débora Machado Nascimento do Espírito Santo ${ }^{1}$, Carina Galvan ${ }^{1}$, Lisiane Paula Sordi Matzenbacher ${ }^{1}$, Rosaura Soares Paczek ${ }^{1 *}$, Ana Karina Silva da Rocha Tanaka², Denise Oliveira D'Avila', Flavia Giendruczak da Silva ${ }^{1}$, Katia Bottega Moraes ${ }^{1}$, Liege Lunardi ${ }^{1}$, Liziane Medianeira Calegari Rigon Gil ${ }^{1}$.

\section{RESUMO}

Objetivo: Descrever como foram implementados e adaptados os leitos de recuperação semi-intensiva, dentro de um Centro Cirúrgico, durante a pandemia da COVID-19. Relato de experiência: Estudo descritivo, realizado em maio de 2021, que relata a experiência de enfermeiras atuantes em um Centro Cirúrgico de um hospital público universitário de grande porte, localizado no Sul do Brasil, sobre as adaptações que ocorreram durante a pandemia da COVID-19. Uma sala cirúrgica foi transformada em leitos de unidade de terapia semiintensiva, auxiliando na recuperação de pacientes cirúrgicos. Diante da avassaladora pandemia, o hospital teve que enfrentar a ausência de recursos disponíveis, estruturando áreas e capacitando funcionários de forma súbita. Considerações finais: Com o agravamento da pandemia, os hospitais são obrigados a tomar medidas drásticas para atender o maior número de pessoas vítimas da COVID-19. Com os leitos de UTI lotados, muitos hospitais vislumbraram o Centro Cirúrgico como sendo um local onde este cuidado complexo pode ser realizado, utilizando a estrutura e os equipamentos desses locais.

Palavras-chave: Cuidados de enfermagem, Cuidados críticos, Cuidados pós-operatórios.

\begin{abstract}
Objective: To describe how semi-intensive recovery beds were implemented and adapted, within an operating room, during the pandemic of COVID-19. Experience report: Descriptive study, carried out in May 2021, which reports the experience of nurses working in a surgical center of a large public university hospital, located in southern Brazil, on the adaptations that occurred during the COVID-19 pandemic. An operating room was transformed into semi-intensive care unit beds, assisting in the recovery of surgical patients. Faced with the overwhelming pandemic, the hospital had to cope with the lack of available resources, structuring areas and training staff suddenly. Final considerations: With the worsening of the pandemic, hospitals are forced to take drastic measures to care for the largest number of people who are victims of COVID-19. With ICU beds crowded, many hospitals have envisioned the surgical center as a place where this complex care can be performed, using the structure and equipment of these places.
\end{abstract}

Keywords: Nursing care, Critical care, Postoperative care.

1 Hospital de Clínicas de Porto Alegre, Porto Alegre - RS. `E-mail: rspaczek@gmail.com
2 Universidade Federal do Rio Grande do Sul, Porto Alegre - RS.

SUBMETIDO EM: 5/2021

ACEITO EM: 6/2021

PUBLICADO EM: 6/2021 


\section{RESUMEN}

Objetivo: Describir cómo se implementaron y adaptaron camas de recuperación semi-intensivas dentro de un centro quirúrgico durante la pandemia del COVID-19. Informe de experiencia: Estudio descriptivo, realizado en mayo de 2021, que relata la experiencia de enfermeras que trabajan en el quirófano de un gran hospital universitario público, ubicado en el sur de Brasil, sobre las adaptaciones ocorridas durante la pandemia del COVID-19. Un quirófano se transformó en camas de unidad de cuidados semi-intensivos, ayudando en la recuperación de los pacientes quirúrgicos. Ante la abrumadora pandemia, el hospital tuvo que enfrentar la falta de recursos disponibles, estructurando áreas y capacitando a los empleados de forma repentina. Consideraciones finales: Con el agravamiento de la pandemia, los hospitales se ven obligados a tomar medidas drásticas para atender a la mayor cantidad de personas que son víctimas del COVID-19. Con las camas de la UCI llenas, muchos hospitales vieron al centro quirúrgico como un lugar donde este complejo de atención puede realizarse, utilizando la estructura y equipamiento de estos lugares.

Palabras clave: Atención de enfermería, Cuidados críticos, Cuidados posoperatorios.

\section{INTRODUÇÃO}

A Coronavirus Disease (COVID-19) é uma doença causada pelo novo Coronavírus que foi identificada na China em dezembro de 2019. Em fevereiro de 2020, no Brasil, já havia o registro de transmissão comunitária, devido à sua elevada transmissibilidade, aliada à ausência de tratamentos reconhecidamente eficazes, que evidenciam as fragilidades hospitalares e, em particular, a falta de profissionais da saúde (OLIVEIRA WK, et al., 2020).

No Centro Cirúrgico (CC) são realizados procedimentos cirúrgicos, diagnósticos e terapêuticos, tanto em caráter eletivo quanto emergencial, onde ocorrem processos complexos, demandando alto investimento para a sua implantação e funcionamento. $O$ ambiente cirúrgico tem a dinâmica do cuidado de Enfermagem voltada à objetividade das ações, cuja intervenção invasiva é de natureza técnica e de recursos materiais com alta complexidade, precisão e eficácia, sendo necessário profissionais habilitados para atender às interfaces atribuídas à dinâmica de trabalho visando à recuperação do paciente (PEDRO DRC, et al., 2018).

Devido às particularidades desse serviço, a interação social no cuidado, muitas vezes, é limitada e, diante desse contexto, os enfermeiros encontram-se com os desafios de organizar as diferentes atribuições que compõem o seu processo de trabalho, implicando o gerenciamento do cuidado de Enfermagem no período transoperatório. Essa condição compreende a conexão entre as dimensões gerencial e assistencial do enfermeiro, como o planejamento e delegações de ações, na previsão e provisão de recursos materiais e humanos, na capacitação de sua equipe de trabalho, objetivando a melhoria dos cuidados e a assistência segura ao paciente (FUGULIN FMT, et al., 2012; SANTOS TBS, et al., 2020).

Com o crescimento da COVID-19, os hospitais apresentaram um desgaste na estrutura de atendimento com sobrecarga para os profissionais da saúde, com elevada demanda emergencial e crescente falta de leitos de Unidade de Tratamento Intensivo (UTI), expondo os sistemas de saúde públicos e privados a uma dimensão mais grave da crise sanitária brasileira: a do seu componente hospitalar. A disposição da estrutura assistencial hospitalar, historicamente insuficiente, fica impactada com essa demanda por um grande número de leitos hospitalares destinados ao cuidado de vítimas do Coronavírus, sobretudo, as mais graves (NORONHA KVMS, et al., 2020; JÚNIOR AMF, et al., 2020; ANDRADE GD, et al., 2021).

Temos no Brasil 11.615 leitos para pacientes adultos em UTI pelo Sistema Único de Saúde (SUS), que não suprem a necessidade e demandam alto custo e complexidade, limitando $O$ atendimento a pacientes graves, podendo comprometer a taxa de mortalidade (MONTEIRO SA, et al., 2020). Pacientes em estado crítico, necessitam de cuidados intensivos por meio de tecnologias e monitoramentos avançados sendo atendidos em UTI, com profissionais especializados, que além da patologia, tratam demandas ambientais, psicossociais e familiares (BARBOSA IEB, 2021; CARVALHO BC, 2019). A atual pandemia do COVID-19 
impôs mudanças na dinâmica da equipe, na organização da carga de trabalho, em medidas do aumento de equipamentos de proteção individual e preparação das instalações (BENÍTEZ CY, et al., 2020).

A cada leito essencial para o atendimento a pacientes COVID-19, são necessários alguns requisitos primordiais, novos fluxos de organização de acesso, novos equipamentos, especialmente os ventiladores mecânicos, bombas de infusão, rede de gases e, notoriamente, profissionais com conhecimento apropriado para atuar na linha de frente (THADEU BSS, et al., 2021).

O objetivo deste estudo é descrever como foram implementados e adaptados os leitos de recuperação semi-intensiva dentro de um CC.

\section{RELATO DE EXPERIÊNCIA}

Trata-se de um estudo descritivo, realizado em maio de 2021, que relata a experiência de enfermeiras atuantes em um centro cirúrgico de um hospital público universitário de grande porte, localizado no Sul do Brasil. O serviço de CC possui treze salas cirúrgicas onde são realizados procedimentos de diversas especialidades e complexidades.

Durante a pandemia foram adotadas diversas medidas, entre elas, o fechamento de algumas salas e abertura de uma sala adequada para $o$ atendimento dos pacientes com a COVID-19. Passou-se a atender em oito salas cirúrgicas, sendo uma sala para atendimento COVID-19, uma sala de urgência, quatro salas para $o$ atendimento a pacientes do SUS, uma sala de convênio e uma sala para cirurgia cardíaca. Nos finais de semana, trabalhou-se com duas salas cirúrgicas, sendo uma para o atendimento de pacientes com COVID19 e outra para as urgências. Os transplantes são atendidos pela equipe durante o dia e à noite e, nos finais de semana, o atendimento é por escala de sobreaviso.

No ano de 2020, houve uma redução significativa no número de cirurgias realizadas devido à pandemia da COVID-19, sendo que as cirurgias eletivas são realizadas de segunda-feira a sábado e as cirurgias de urgências são realizadas nas 24 horas do dia. O quantitativo dos profissionais de Enfermagem atuantes no CC são: 15 enfermeiras e 105 técnicos de Enfermagem.

Com o alto índice de ocupação de leitos de UTI por pacientes infectados com a COVID-19 e a necessidade de um número maior de leitos para o atendimento a pacientes críticos e oncológicos, as cirurgias eletivas foram canceladas, reduzindo a demanda de trabalho no CC. Existe uma demanda de procedimentos cirúrgicos oncológicos com uma grande lista de espera, impactando a saúde dos pacientes, tornando essas cirurgias uma urgência. Algumas estratégias foram elaboradas disponibilizando leitos para prestar assistência a esses casos, e uma sala cirúrgica do CC foi adaptada em dois leitos de UTI semi-intensiva.

A mudança da sala cirúrgica para dois leitos semicríticos desenvolveu-se após a aprovação do comitê de crise do hospital, visando ao atendimento dos pacientes pós-cirúrgicos que necessitavam de cuidados semiintensivos, sendo avaliado diariamente cada caso conforme a necessidade de ocupação desses leitos, visto que o hospital estava passando por um momento crítico de lotação.

Conforme a experiência das áreas críticas do hospital, os leitos foram montados observando a distribuição do espaço físico da sala, os aparelhos de anestesia, a instalação de gases e as medicações para o pronto uso, tendo o envolvimento da equipe de Enfermagem, do técnico da Engenharia Clínica, da farmacêutica da unidade e do serviço de anestesia. Os equipamentos utilizados foram da própria área cirúrgica. Com a diminuição das cirurgias, materiais foram aproveitados (bombas de infusão, pressurizadores, caixas para medicações) e as camas foram emprestadas de outros setores. A sala foi estruturada por duas enfermeiras do turno da tarde do $\mathrm{CC}$, em três dias, com o apoio de dois técnicos de Enfermagem do turno da tarde e um da noite. Para essa conversão, foi instalado um computador e uma impressora especificamente para essa sala para imprimir as prescrições médicas e de Enfermagem.

Foram montados diversos kits para facilitar o trabalho diário da equipe de Enfermagem, como: kit de Pressão Arterial Média (PAM), contendo todo o material necessário para a instalação ou punção de uma PAM; 
kit curativo; kit via aérea (máscaras de Hudson, de Venturi e todo o material para uma possível intubação traqueal); kit sondagem vesical e kit banho. Na cabeceira dos leitos, havia uma caixa contendo todo o material necessário para a instalação de oxigênio. Nas paredes da sala, foram colocados cartazes orientando quanto à interação medicamentosa com o intuito de facilitar e reduzir os erros no preparo e na administração de medicamentos.

Também foi elaborada uma pasta de apoio para a Enfermagem com toda a parte teórica da capacitação realizada para a equipe, os protocolos descritos dessa UTI intermediária e o quantitativo de materiais que havia dentro dessa sala para um controle diário. Ocorreram a contratação de médicos para os atendimentos desses pacientes e o direcionamento de enfermeiros para a supervisão e o atendimento junto aos técnicos de Enfermagem.

Foram realizadas capacitações ministradas por duas enfermeiras da área cirúrgica, como também a estruturação de protocolos de atendimento, garantindo a qualidade e a segurança dos cuidados realizados pela equipe de Enfermagem nos pacientes semicríticos no pós-operatório imediato, tendo como apoio, também, os treinamentos à distância sobre pacientes críticos e reuniões para o alinhamento do processo de desenvolvimento desse projeto dentro da área do CC.

Houve uma grande preocupação com a especificidade do manejo desses pacientes, pois a equipe do CC não possui prática em cuidados a pacientes críticos no pós-operatório. Assim, surgiu a necessidade de iniciar um treinamento para toda a equipe. Foram realizadas as capacitações teóricas com duas horas de duração, assim como os treinamentos práticos no setor responsável pela educação continuada do hospital, dando enfoque especial à diluição de medicamentos, à aspiração de pacientes em uso de tubo orotraqueal e aos cuidados específicos com cateteres e sondas. Concomitantemente, deslocaram-se seis enfermeiros e vinte técnicos de Enfermagem envolvendo todos os turnos de trabalho para a capacitação na prática assistencial dentro da UTI, localizada em frente ao CC.

As capacitações práticas foram realizadas durante a jornada de trabalho desses profissionais, desempenhando uma abordagem e a revisão de rotinas como a frequência da verificação de sinais vitais, assim como o balanço hídrico, as drogas comumente utilizadas em UTI com os seus efeitos, as diluições e interações medicamentosas, a revisão da técnica de instalação de pressão arterial invasiva, a pressão venosa central, os cuidados com a Derivação Ventricular Externa (DVE) e a prescrição de Enfermagem com os diagnósticos específicos de cuidados intensivos. Com a finalidade de auxiliar os enfermeiros, foram elaborados, também, vídeos explicativos demonstrando quanto à medida da PAM e ao detalhamento das prescrições de Enfermagem.

A equipe para o atendimento foi composta por uma enfermeira do CC, dois técnicos de Enfermagem e um anestesista para cada turno de trabalho, formando uma equipe especializada para $o$ atendimento aos pacientes que seriam designados para a recuperação em leitos de terapia semi-intensiva.

As cirurgias determinadas para que a recuperação fosse realizada na sala da UTI semi-intensiva dentro do CC foram escolhidas pelo colegiado do hospital, com uma semana de antecedência, conforme o mapa cirúrgico pré-agendado. Os procedimentos cirúrgicos elencados foram: retossigmoidectomia; prostatectomia; colectomia; bypass fêmoro-poplíteo; endarterectomia de carótida; duodenopancreatectomia e esofagectomia. As cirurgias eram deliberadas pelo porte cirúrgico, gravidade, avanço da doença e a necessidade de suporte assistencial estendido no pós-operatório como pernoite em unidade de terapia semi-intensiva.

O objetivo desses leitos é prestar assistência ao paciente enfermo, na sua grande maioria, oncológico, que está em lista de espera pelo SUS e que, em virtude da pandemia da COVID-19, teve o seu procedimento cirúrgico adiado. Devido ao afastamento dos pacientes aos tratamentos adequados, o avanço da patologia pode ter consequências substanciais na vida do indivíduo como um todo, levando ao óbito precoce.

Diante dessa realidade imposta durante a pandemia, o envolvimento de diferentes áreas e profissionais refletiu no planejamento das ações realizadas, demonstrando espaços para a discussão das necessidades, dos obstáculos do ser enfermeiro, do reconhecimento da Enfermagem e do protagonismo da profissão. 


\section{DISCUSSÃO}

Com as Unidades de Terapia Intensiva (UTIs) da instituição lotadas em razão da pandemia, houve a necessidade de um número maior de leitos para o atendimento a pacientes críticos e oncológicos. Até mesmo as equipes mais experientes vivem agora em meio a circunstâncias delicadas e restritas, em que é preciso se adaptar para atender os pacientes com segurança e qualidade. Setores como os CCs, as salas de recuperação, os leitos de emergência, os leitos clínicos e de enfermaria transformaram-se em UTIs, mesmo aqueles setores que foram planejados para atender os pacientes de menor gravidade.

O esgotamento de vagas nas UTIs das redes pública e privada impacta todos os demais procedimentos. Quem não está infectado, mas enfrenta um problema de saúde também, infelizmente, acaba prejudicado, já que as cirurgias eletivas foram suspensas para garantir leitos a quem está infectado, uma vez que a doença avança rapidamente e a permanência na UTI é prolongada.

Diante da precariedade de recursos físicos, estruturais e humanos, surge a questão sobre o seu uso e alocamento de forma justa. O Conselho Federal de Medicina (CFM) elaborou a Resolução $n^{\circ}$ 2.156/2016, onde o critério para ocupação de leitos de UTI é a instabilidade crítica. Esta resolução estabelece níveis de prioridade. Apresenta-se como uma alternativa na distribuição de leitos a possibilidade de padronização desses parâmetros pela Administração Pública na esfera da União (MOREIRA RS, 2020).

Devido à pandemia da COVID-19, os critérios de admissão em leitos de UTI são complexos e desafiadores, já que os pacientes não admitidos em UTI apresentam taxa de mortalidade elevada. Esta seleção de ocupação dos leitos tem se tornado um assunto relevante pela perspectiva de assegurar vagas a pacientes com elevadas chances de recuperação. É crucial a adoção de medidas urgentes para otimizar o uso dos serviços públicos e privados existentes, bem como de investimentos para aumentar a capacidade de resposta do sistema de saúde nas regiões de maior necessidade (RACHE B, et al., 2020; MENDES FF, 2020).

Encontram-se várias fragilidades na disponibilização de recursos, tais como: baixo quantitativo de recursos humanos, principalmente capacitados para prestar cuidados em áreas com paciente crítico; leitos gerais e de UTI, assim como os equipamentos de ventilação assistida. A falta de critérios estabelecidos para a alocação assertiva desses recursos é notória. Os dados apontam a necessidade de gestores em saúde, assim como de profissionais da área estarem preparados para o uso adequado dos recursos humanos e materiais, otimizando, dessa forma, os escassos meios existentes (SILVA KR, et al., 2020).

Enfatiza-se, atentando a urgência das decisões e ações a serem realizadas nesse cenário de pandemia, a atuação dos gestores e profissionais da saúde embasados e alicerçados em evidências científicas e recomendações dos órgãos competentes nos âmbitos mundial e nacional para a estruturação de unidades, abstendo-se de improvisos e condutas provisórias. O enfermeiro assume papel fundamental na estruturação e organização de áreas físicas, assim como na gestão de recursos humanos capacitados (BITENCOURT JVOV, et al., 2020).

A pandemia da COVID-19 evidencia, como protagonistas, os profissionais da Enfermagem pelo maior aproveitamento de enfermeiros e anestesistas no gerenciamento dos casos críticos, visto terem especialidades profissionais próprias, como o manejo das vias aéreas, incluindo os ventiladores e o uso de equipamentos de oxigênio de alto fluxo, fundamentais no manejo de pacientes com COVID-19. Contudo, como a rotina desses profissionais geralmente limita-se aos centros cirúrgicos, necessitam de capacitação para realizar procedimentos de Enfermagem regulares em UTI (RIBEIRO JR, et al., 2021).

Com o agravo da pandemia, os hospitais são obrigados a tomar medidas drásticas para atender o maior número de pessoas vítimas da COVID-19, com UTIs lotadas, uma alternativa é o uso do CC para atendimento a pacientes críticos em pós operatório. Uma nova rotina foi criada, procedimentos assistenciais foram regularizados, capacitações e treinamentos das equipes foram essenciais para o sucesso dessa mudança. Essas reflexões contribuem para propiciar aos enfermeiros gestores, a superação dos desafios e a implementação de estratégias alicerçadas no conhecimento científico e nos padrões de qualidade e segurança assistencial. 


\section{REFERÊNCIAS}

1. ANDRADE GD, et al. Residência multiprofissional em unidade de terapia intensiva: experiências exitosas em tempos de pandemia. Revista Eletrônica Acervo Saúde, 2021; 13(4).

2. BARBOSA IEB, et al. Fatores que difundem a assistência de enfermagem humanizada na unidade de terapia intensiva. Revista Eletrônica Acervo Saúde, 2021; 13(4).

3. BENÍTEZ CY, et al. Adaptação a um cenário sem precedentes: cirurgia durante o surto de COVID-19. Rev Col Bras Cir, 2020; 47: e20202701.

4. BITENCOURT JVOV, et al. Protagonismo do enfermeiro na estruturação e gestão de uma unidade específica para Covid-19. Texto \& contexto enferm. (Online), 2020; 29: e20200213.

5. CARVALHO BC. Atuação da equipe de enfermagem no cuidado ao paciente grave. Revista Eletrônica Acervo Saúde, 2019; 17: e36.

6. FUGULIN FMT, et al. Tempo de assistência de Enfermagem em unidade de terapia intensiva: avaliação dos parâmetros propostos pela Resolução COFEN no293/04. Rev. latinoam. enferm. (Online), 2012; 20(2).

7. HAUSMANN M, PEDUZZI M. Articulação entre as dimensões gerencial e assistencial do processo de trabalho do enfermeiro. Texto \& contexto enferm. (Online), 2009; 18(2): 258-65.

8. JÚNIOR AMF, et al. COVID-19 em profissionais da saúde, vivências e perspectivas: um relato de experiência. Revista Eletrônica Acervo Saúde, 2020; 12(12): e5258.

9. MENDES FF. COVID-19 e a retomada das cirurgias eletivas. Como voltaremos à normalidade? Rev. bras. anestesiol., 2020; 70(5): 455-6.

10. MONTEIRO SA, et al. Aplicação do nursing activities score nas unidades de terapia intensiva brasileira: revisão integrativa da literatura. Revista Eletrônica Acervo Saúde, 2020; 12(10).

11. MOREIRA RS. COVID-19: unidades de terapia intensiva, ventiladores mecânicos e perfis latentes de mortalidade associados à letalidade no Brasil.Cad. Saúde Pública, 2020; 36(5)

12. NORONHA KVMS, et al. Pandemia por COVID-19 no Brasil: análise da demanda e da oferta de leitos hospitalares e equipamentos de ventilação assistida segundo diferentes cenários. Cad. Saúde Pública, 2020; 36(6): e00115320.

13. OLIVEIRA WK, et al. Como o Brasil pode deter a COVID-19. Epidemiologia e Serviços de Saúde, 2020; 29(2): e2020044.

14. PEDRO DRC, et al. Dimensionamento do pessoal de enfermagem em centro cirúrgico de um hospital universitário. J Nurs Health. 2018; 8(1): e188108.

15. RACHE B, et al. Necessidades de Infraestrutura do SUS em Preparo à COVID-19: Leitos de UTI, Respiradores e Ocupação Hospitalar. Nota Técnica n.3. 2020; IEPS: São Paulo unidade específica para Covid-19. Texto \& contexto enferm. (Online), 2020; 29: e20200213.

16. RIBEIRO JR, et al. Profissionais de Enfermagem na UTI e seu protagonismo na pandemia: Legados da Covid-19. Revista Enfermagem Contemporânea, 2021; 10(2).

17. SANTOS TBS, et al. Gestão hospitalar no Sistema Único de Saúde: problemáticas de estudos em política, planejamento e gestão em saúde. Ciência \& Saúde Coletiva, 2020; 25: 3597-3609.

18. SILVA KR, et al. Alocação de recursos para assistência à saúde em tempos da pandemia de COVID-19: revisão integrativa. Rev. Bras. Enferm., 2020; 73(2): e20200244 